\title{
The Existence and Application of Unbounded Connected Components
}

\author{
Hua Luo ${ }^{1}$ and Ruyun $\mathrm{Ma}^{2}$ \\ ${ }^{1}$ School of Mathematics and Quantitative Economics, Dongbei University of Finance and Economics, Dalian 116025, China \\ ${ }^{2}$ Department of Mathematics, Northwest Normal University, Lanzhou 730070, China \\ Correspondence should be addressed to Hua Luo; luohuadufe@163.com
}

Received 25 December 2013; Accepted 5 March 2014; Published 6 April 2014

Academic Editor: Bo-Qing Dong

Copyright (c) $2014 \mathrm{H}$. Luo and R. Ma. This is an open access article distributed under the Creative Commons Attribution License, which permits unrestricted use, distribution, and reproduction in any medium, provided the original work is properly cited.

Let $X$ be a Banach space and $C_{n}$ a family of connected subsets of $R \times X$. We prove the existence of unbounded components in superior limit of $\left\{C_{n}\right\}$, denoted by $\overline{\lim } C_{n}$, which have prescribed shapes. As applications, we investigate the global behavior of the set of positive periodic solutions to nonlinear first-order differential equations with delay, which can be used for modeling physiological processes.

\section{Introduction and the Main Results}

The connectivity result on the fixed set of a 1-parameter family of maps, which goes back to Leray and Schauder [1] and was proved in its full generality by Browder [2], is a useful tool in the study of global continua of solutions on nonlinear differential equations. Costa and Gonçalves [3] stated and proved a suitable version for the study of nonlinear boundary value problems at resonance. Massabò and Pejsachowicz [4] generalized the main results of $[1,2]$ to the $n$-parameter family of compact vector fields. The above results were established when the parameter(s) changes in a bounded set. Sun and Song [5] proved the existence of unbounded connected component of 1-parameter family of compact vector fields, where the parameter varies on whole real line. All of these results play important roles in the study of nonlinear functional analysis and nonlinear differential equations.

For clearly reading, we firstly recall Kuratowski's definitions and notations in [6].

Let $\mathbb{M}$ be a metric space. Let $\left\{C_{n} \mid n=1,2, \ldots\right\}$ be a family of subsets of $\mathbb{M}$. Then the superior limit $\mathscr{D}$ of $\left\{C_{n}\right\}$ is defined by

$$
\begin{array}{r}
\mathscr{D}:=\varlimsup \\
\lim C_{n}=\left\{x \in M \mid \exists\left\{n_{i}\right\} \subset \mathbb{N}, x_{n_{i}} \in C_{n_{i}},\right. \\
\text { such that } \left.x_{n_{i}} \longrightarrow x\right\} .
\end{array}
$$

A component of a set $\mathbb{M}$ means a maximal connected subset of $\mathbb{M}$.

Definition 1. Let $X$ be a Banach space with the norm $\|\cdot\|$. Let $\zeta$ be a component of solutions in $\mathbb{R} \times X$. $\zeta$ meets $(a, \mathbf{0})$ and infinity means that there existed a sequence $\left\{\left(\lambda_{k}, u_{k}\right)\right\} \subset$ $[\zeta \backslash\{(a, 0)\}]$ such that $\left(\lambda_{k}, u_{k}\right) \rightarrow(a, 0)$ as $k \rightarrow \infty$.

For $\rho, \beta \in(0, \infty)$, let us denote

$$
\begin{gathered}
B_{\rho}:=\{u \in X \mid\|u\| \leq \rho\}, \\
\Omega_{\beta, \rho}:=([0, \infty) \times X) \backslash\{(\eta, u) \in[\beta, \infty) \times X \mid\|u\| \leq \rho\} .
\end{gathered}
$$

Let $\left\{\mathscr{C}_{n}\right\}$ be a family of connected subsets of $\mathbb{R} \times X$. The purpose of this paper is to study the existence of unbounded components in $\overline{\lim } \mathscr{C}_{n}$ which have prescribed shapes.

More precisely, we will prove the following theorems.

Theorem 2. Let $X$ be a Banach space and let $\left\{\mathscr{C}_{n}\right\}$ be a family of connected subsets of $[0, \infty) \times X$. Assume that

(A1) there exist $0<\sigma<r<\infty$ and $\lambda_{*} \in(0, \infty)$, such that

$$
\mathscr{C}_{n} \cap\left\{(\mu, y) \mid 0<\mu \leq \lambda_{*}+\sigma, r-\sigma \leq\|y\| \leq r+\sigma\right\}=\emptyset \text {; }
$$

(A2) $\eta_{n} \rightarrow 0^{+}$and $\mathscr{C}_{n}$ meets $\left(\eta_{n}, \mathbf{0}\right)$ and infinity; 
(A3) for every $R>0,\left(\bigcup_{n=1}^{\infty} \mathscr{C}_{n}\right) \cap \mathbb{B}_{R}$ is a relatively compact set of $\mathbb{R} \times X$, where

$$
\mathbb{B}_{R}=\{(\mu, x) \in \mathbb{R} \times X|| \mu \mid<R,\|x\| \leq R\} .
$$

Then there exists a component $\mathscr{C}$ in $\overline{\lim } \mathscr{C}_{n}$ satisfying

(a) $\mathscr{C}$ meets $(0,0)$ and infinity;

(b) $\mathscr{C} \cap\left\{(\mu, y) \mid 0<\mu \leq \lambda_{*},\|y\|=r\right\}=\emptyset$.

Theorem 3. Let $a \in \mathbb{R}$ be a constant. Let $X$ be a Banach space, and let $\left\{\mathscr{C}_{n}\right\}$ be a family of connected subsets of $\mathbb{R} \times X$. Assume that

(H1) $\mathscr{C}_{n} \cap((-\infty, a] \times X)=\emptyset$;

(H2) there exist $0<\sigma<r<\infty$ and $b \in(a, \infty)$, such that

$\mathscr{C}_{n} \cap\{(\mu, y) \mid \mu \geq b-\sigma, r-\sigma \leq\|y\| \leq r+\sigma\}=\emptyset ;$

(H3) $\eta_{k}>$ a for all $k \in \mathbb{N}, \eta_{k} \rightarrow+\infty$ and $\mathscr{C}_{n}$ meets $\left(\eta_{n}, \mathbf{0}\right)$ and infinity in $([a, \infty) \times X) \backslash \Omega_{b, r}$;

(H4) for every $R>0,\left(\bigcup_{n=1}^{\infty} \mathscr{C}_{n}\right) \cap \mathbb{B}_{R}$ is a relatively compact set of $\mathbb{R} \times X$.

Then there exists a component $\mathscr{C}$ in $\overline{\lim } \mathscr{C}_{n}$ such that

(a) both $\mathscr{C} \cap \Omega_{b, r}$ and $\mathscr{C} \cap\left(([a, \infty) \times X) \backslash \Omega_{b, r}\right)$ are unbounded;

(b) $\mathscr{C} \cap\{(\mu, y) \mid \mu \geq b,\|y\|=r\}=\emptyset$.

\section{Proofs of the Main Results}

To prove Theorems 2 and 3, we need the following preliminary result, which is proved by $\mathrm{Ma}$ and An [7] by using Whyburn lemma, and the method of Sun and Song to prove [6, Lemma 2.2].

Lemma 4 (see [7, Lemma 2.2]). Let $E$ be a Banach space with the norm $\|\cdot\|_{E}$. Let $\left\{D_{n}\right\}$ be a family of connected subsets of $E$. Assume that

(i) there exist $z_{n} \in D_{n}, n=1,2, \ldots$, and $z^{*} \in E$, such that $z_{n} \rightarrow z^{*}$

(ii) $\lim _{n \rightarrow \infty} r_{n}=\infty$, where $r_{n}=\sup \left\{\|x\|_{E} \mid x \in D_{n}\right\}$;

(iii) for every $R>0,\left(\bigcup_{n=1}^{\infty} D_{n}\right) \cap B_{R}$ is a relatively compact set of $E$, where

$$
B_{R}=\left\{x \in E \mid\|x\|_{E} \leq R\right\} .
$$

Then there exists an unbounded component $\mathscr{C}$ in $\overline{\lim } D_{n}$ and $z^{*} \in \mathscr{C}$.

Proof of Theorem 2. (a) It is a direct consequence of Lemma 4.

(b) Assume, on the contrary, that the conclusion is not true. Then there exists $\left(\mu^{*}, u^{*}\right) \in \mathscr{C}$ with $\mu^{*} \leq \lambda_{*}$ and $\left\|u^{*}\right\|=$ $r$. Hence, there exists $\left\{\left(\eta_{n_{k}}, u_{n_{k}}\right)\right\} \subset \mathscr{C}_{n}$, such that

$$
\lim _{k \rightarrow \infty} \eta_{n_{k}}=\mu^{*}, \quad \lim _{k \rightarrow \infty} u_{n_{k}}=u^{*} .
$$

Thus, there exists $k_{0} \in \mathbb{N}$, such that, for $k \geq k_{0}$,

$$
\eta_{n_{k}}<\lambda^{*}+\frac{\sigma}{2}, \quad r-\frac{\sigma}{2} \leq\left\|u_{n_{k}}\right\| \leq r+\frac{\sigma}{2} .
$$

However, this contradicts (3).

Proof of Theorem 3. (a) Since $\eta_{k} \rightarrow \infty$, we may assume that

$$
\eta_{k}>b, \quad k \in \mathbb{N} \text {. }
$$

So, it follows from conditions (ii) and (iii) that $\mathscr{C}_{n}$ meets $\{b\} \times$ $B_{r}$ and infinity in $([a, \infty) \times X) \backslash \Omega_{b, r}$.

For each $(b, v) \in\left(\{b\} \times B_{r}\right) \cap \mathscr{D}$, let $\mathscr{E}(b, v)(\subset \mathscr{D})$ be a component containing $(b, v)$. Let

$$
\varsigma(b, v):=\sup \left\{\lambda \mid(\lambda, u) \in \mathscr{E}(b, v), u \in B_{r}\right\} .
$$

Set

$$
\begin{aligned}
\Pi:=\{(b, v) \mid & (b, v) \in\left(\{b\} \times B_{r}\right) \cap \mathscr{D}, \mathscr{E}(b, v) \\
& \text { is unbounded in } \left.([a, \infty) \times X) \backslash \Omega_{b, r}\right\} .
\end{aligned}
$$

Then $\Pi \neq \emptyset$ since

$$
\left(\mathscr{C}_{j} \cap\left(\{b\} \times B_{r}\right)\right) \subseteq \Pi, \quad j \in \mathbb{N} .
$$

From Lemma 4 , it follows that $\Pi$ is closed in $[0, \infty) \times X$, and, furthermore, $\Pi$ is compact in $[0, \infty) \times X$.

Let

$$
\Sigma^{\diamond}:=\bigcup_{(b, v) \in \Pi} \mathscr{E}(b, v)
$$

By Lemma $4, \overline{\lim } C_{n} \cap\left(([a, \infty) \times X) \backslash \Omega_{b, r}\right)$ contains a component $\zeta$ which meets $\{b\} \times B_{r}$ and infinity in $(([a, \infty) \times$ $\left.X) \backslash \Omega_{b, r}\right)$. Obviously

$$
\zeta \in \Sigma^{\diamond} \text {. }
$$
holds.

If $\varsigma(b, v)=+\infty$ for some $(b, v) \in \Pi$, then Theorem 3

Assume, on the contrary, that $\varsigma(b, v)<+\infty$ for all $(b, v) \in$ $\Pi$.

For every $(b, v) \in \Pi$, let $\mathscr{E}^{\triangleright}(b, v)$ be the component in $\mathscr{E}(b, v) \cap\left([b, \infty) \times B_{r}\right)$ which contains $(b, v)$. Using the standard method, we can find a bounded open set $U(b, v)$ in $[b, \infty) \times B_{r}$, such that

$$
\mathscr{E}^{\triangleright}(b, v) \subset U(b, v), \quad \partial U(v) \cap \Sigma^{\diamond}=\emptyset
$$

and for every $(b, v) \in \Pi$,

$$
\sup \{\lambda \mid(\lambda, u) \in \bar{U}(b, v)\}<\infty,
$$

where $\partial U(v)$ and $\bar{U}(v)$ are the boundary and closure of $U(v)$ in $[b, \infty) \times B_{r}$, respectively.

Evidently, the following family of the open sets of $\{b\} \times B_{r}$

$$
\left\{U(b, v) \cap\left(\{b\} \times B_{r}\right) \mid(b, v) \in \Pi\right\}
$$


is an open covering of $\Pi$. Since $\Pi$ is compact set in $\{b\} \times B_{r}$, there exist $v_{1}, \ldots, v_{m}$ such that $\left(b, v_{i}\right) \in \Pi(i=1, \ldots, m)$, and the family of open sets in $\{b\} \times B_{r}$ :

$$
\left\{U\left(b, v_{i}\right) \cap\left(\{b\} \times B_{r}\right) \mid i=1, \ldots, m\right\}
$$

is a finite open covering of $\Pi$. This implies that

$$
\Pi \subseteq\left\{U\left(b, v_{i}\right) \cap\left(\{b\} \times B_{r}\right) \mid i=1, \ldots, m\right\} .
$$

Let

$$
U_{1}=\bigcup_{i=1}^{m} U\left(b, v_{i}\right)
$$

Then $U_{1}$ is a bounded open set in $[b, \infty) \times B_{r}$,

$$
\partial U_{1} \cap\left(\bigcup_{(b, v) \in \Pi} \mathscr{E}^{\triangleright}(b, v)\right)=\emptyset, \quad \partial U_{1} \cap \Sigma^{\diamond}=\emptyset
$$

and by (16), we have

$$
\sup \left\{\lambda \mid(\lambda, u) \in \bar{U}_{1}\right\}<\infty
$$

where $\partial U_{1}$ and $\bar{U}_{1}$ are the boundary and closure of $U_{1}$ in $[b, \infty) \times B_{r}$, respectively.

Now, (22) together with (19) and (21) implied that

$$
\sup \left\{\lambda \mid(\lambda, u) \in \Sigma^{\diamond}, u \in B_{r}\right\}<\infty .
$$

However, this contradicts $\lambda_{n} \rightarrow \infty$.

Therefore, there exists $\left(b, v^{*}\right) \in \Pi$ such that $\zeta:=\mathscr{E}\left(b, v^{*}\right)$ which is unbounded in both $[b, \infty) \times B_{r}$ and $([a, \infty) \times X) \backslash$ $\left([b, \infty) \times B_{r}\right)$.

(b) By a fully analogous argument as in the proof of Theorem 2(b) (with minor modifications), one can immediately obtain the desired results.

\section{Application to Functional Differential Equations}

In recent years, there has been considerable interest in the existence of $\omega$-periodic solutions of the equation

$$
u^{\prime}(t)=a(t) g(u(t)) u(t)-\lambda h(t) f(u(t-\tau(t))), \quad t \in \mathbb{R},
$$

where $a, h \in C(\mathbb{R},[0, \infty))$ are $\omega$-periodic functions and $\tau$ is a continuous $\omega$-periodic function. Equation (24) has been proposed as a model for a variety of physiological processes and conditions including production of blood cells, respiration, and cardiac arrhythmias. See, for example, [8-20] and the references therein.

Recently, Wang [18] used the fixed point index [20, 21] to study the existence, multiplicity, and nonexistence of positive solutions of (24) under the following assumptions.

(C1) $a, h \in C(\mathbb{R},[0, \infty))$ are $\omega$-periodic functions, $\int_{0}^{\omega} a(t)$ $d t>0, h(t)>0$ on $[0, \omega] ; \tau \in C(\mathbb{R}, \mathbb{R})$ is $\omega$-periodic functions.
(C2) $f, g:[0, \infty) \rightarrow[0, \infty)$ are continuous. $0<l \leq$ $g(s) \leq L$ for $s>0, l, L$ are given positive constants. $f(s)>0$ for $s>0$.

Let $\sigma:=e^{-\int_{0}^{\omega} a(t) d t}$ and denote

$$
\begin{aligned}
& M(r):=\max _{0 \leq t \leq r}\{f(t)\}, \\
& m(r):=\min \left\{f(t): \frac{\sigma^{L}\left(1-\sigma^{l}\right)}{1-\sigma^{L}} r \leq t \leq r\right\} ; \\
& f_{0}:=\lim _{u \rightarrow 0^{+}} \frac{f(u)}{u}, \quad f_{\infty}:=\lim _{u \rightarrow+\infty} \frac{f(u)}{u} .
\end{aligned}
$$

His results provide no any information about the global behavior of the set of positive solutions of (24).

In this section, we will use Theorems 2 and 3 to establish several results on the global behavior of the set of positive solutions of (24), and, accordingly, we get some existence and multiplicity results of positive solutions of (24).

We will work essentially in the Banach space $X=\{u \in$ $C[0, \omega] \mid u$ is $\omega$-periodic. $\}$ with sup norm $\|\cdot\|$.

By a positive solution of $(24)$, we mean a pair $(\lambda, u)$, where $\lambda>0$ and $u$ is a solution of (24) with $u>0$ on $[0, \omega]$.

Let $\Sigma \subset \mathbb{R}^{+} \times E$ be the closure of the set of positive solutions of $(24)$.

We extend the function $f$ to a continuous function $\tilde{f}$ defined on $\mathbb{R}$ in such a way that $\tilde{f}>0$ for all $s<0$. For $\lambda>0$, we then look at arbitrary solutions $u$ of the eigenvalue problem

$$
\begin{array}{r}
-u^{\prime}(t)+a(t) g(u(t)) u(t)=\lambda h(t) \tilde{f}(u(t-\tau(t))), \\
t \in \mathbb{R}, u \text { is } \omega \text {-periodic. }
\end{array}
$$

It was shown in [18] that (26) is equivalent to

$$
u(t)=\lambda \int_{t}^{t+\omega} G_{u}(t, s) h(s) \tilde{f}(u(s-\tau(s))) d s,
$$

where

$$
G_{u}(t, s)=\frac{e^{-\int_{t}^{s} a(\theta) g(u(\theta)) d \theta}}{1-e^{-\int_{0}^{\omega} a(\theta) g(u(\theta)) d \theta}}, \quad t \leq s \leq t+\omega .
$$

By the positivity of Green's function $G_{u}(\cdot, \cdot), h(\cdot)$, and $f(\cdot)$, such solutions are positive. Therefore, the closure of the set of nontrivial solutions $(\lambda, u)$ of $(24)$ in $\mathbb{R}^{+} \times X$ is exactly $\Sigma$.

Next, we consider the spectrum of the linear eigenvalue problem

$$
\begin{array}{r}
-u^{\prime}(t)+a(t) c u(t)=\lambda h(t) u(t-\tau(t)), \\
t \in \mathbb{R}, u \text { is } \omega \text {-periodic. }
\end{array}
$$

Lemma 5. Let c be a positive constant. Then the linear problem (29) has a unique eigenvalue $\lambda^{\diamond}(c)$, which is positive and simple, and the corresponding eigenfunction $\psi(\cdot)$ is of one sign.

Proof. Define an operator $T: X \rightarrow X$ by

$$
T u(t)=\lambda \int_{t}^{t+\omega} G_{u}(t, s) h(s) \tilde{f}(u(s-\tau(s))) d s .
$$


Let $K$ be the cone

$$
K=\{u \in X \mid u \geq 0\}
$$

Then it follows from [18, Lemma 2.2] that $T: K \rightarrow$ $K$ is strongly positive and completely continuous. Thus, the desired result is a direct consequence of Krein-Rutman Theorem, cf. [22, Theorem 19.3].

Theorem 6. Let (C1)-(C2) hold. Assume that

(C3) $f_{0}=\infty=f_{\infty}$.

Then $\Sigma$ contains a component $\mathscr{C}$ satisfying

(1) $\mathscr{C}$ meets $(0,0)$ and $(0, \infty)$;

(2) for every $r>0$, there exists $\mu(r)>0$, such that

$$
\mathscr{C} \cap\{(\mu, u) \in(0, \mu(r)) \times X \mid\|u\|=r\}=\emptyset .
$$

Corollary 7. Let (C1)-(C3) hold. Then there is a constant $\eta_{*}>$ 0 such that (24) has at least two positive solutions as $0<\lambda<$ $\eta_{*}$.

Proof. Let

$$
\begin{aligned}
& B:=\sup \{\lambda \mid(\lambda, u) \in \mathscr{C}\}, \\
& \eta_{*}:=\sup \{\mu(r) \mid r \in(0, \infty)\} .
\end{aligned}
$$

Then $0<\eta_{*} \leq B$. It is easy to see from Theorem 6 that (24) has at least two positive solutions as $0<\lambda<\eta_{*}$.

Denote the cone $K$ in $X$ by

$$
\begin{gathered}
K=\{u \in X \mid u(t) \geq 0 \text { on }[0, \omega], u(t) \geq \delta\|u\|\}, \\
\delta:=\frac{\sigma^{L}\left(1-\sigma^{l}\right)}{1-\sigma^{L}},
\end{gathered}
$$

and for $r>0$, let

$$
K_{r}=\{u \in K \mid\|u\|<r\} .
$$

Define an operator $T_{\lambda}: \quad K \rightarrow X$ by

$$
\begin{array}{r}
T_{\lambda} u(t)=\lambda \int_{t}^{t+\omega} G_{u}(t, s) h(s) \tilde{f}(u(s-\tau(s))) d s, \\
t \in[0, \omega] .
\end{array}
$$

Lemma 8 (see [18]). Assume that (C1)-(C2) hold. Then $T_{\lambda}$ : $K \rightarrow K$ is completely continuous.

Lemma 9. Let (C1)-(C2) hold. If $u \in \partial K_{r}, r>0$, then

$$
\left\|T_{\lambda} u\right\| \leq \lambda \frac{M_{r}}{1-\sigma^{l}} \int_{0}^{\omega} h(s) d s
$$

where $M_{r}=1+\max _{\delta r \leq s \leq r}\{f(s)\}$.
Proof. It is well known from Wang [18] that

$$
\frac{\sigma^{L}}{1-\sigma^{L}} \leq G_{u}(t, s) \leq \frac{1}{1-\sigma^{l}}, \quad t \leq s \leq t+\omega .
$$

This together with the fact that $h$ is $\omega$-periodic and $u(t) \geq \delta r$ on $[0, \omega]$ implies that

$$
\left\|T_{\lambda} u\right\| \leq \lambda \frac{M_{r}}{1-\sigma^{l}} \int_{t}^{t+\omega} h(s) d s=\lambda \frac{M_{r}}{1-\sigma^{l}} \int_{0}^{\omega} h(s) d s .
$$

To prove above Theorem 6 , we define $f^{[n]}:[0, \infty) \rightarrow$ $[0, \infty)$ by

$$
f^{[n]}(s)= \begin{cases}f(s), & s \in\left(\frac{1}{n}, \infty\right), \\ n f\left(\frac{1}{n}\right) s, & s \in\left[0, \frac{1}{n}\right] .\end{cases}
$$

Then $f^{[n]} \in C([0, \infty),[0, \infty))$ with

$$
f^{[n]}(s)>0, \quad \forall s \in(0, \infty), \quad\left(f^{[n]}\right)_{0}=n f\left(\frac{1}{n}\right)>0 .
$$

By (C3), it follows that

$$
\lim _{n \rightarrow \infty}\left(f^{[n]}\right)_{0}=\infty
$$

Now let us consider the auxiliary family of the equations

$$
u^{\prime}(t)=a(t) g(u(t)) u(t)-\lambda h(t) f^{[n]}(u(t-\tau(t))),
$$
$m t \in \mathbb{R}, u$ is $\omega$-periodic.

Let $\xi, \chi \in C[0, \infty)$ be such that

$$
\begin{aligned}
f^{[n]}(s) & =\left(f^{[n]}\right)_{0} s+\xi(s)=n f\left(\frac{1}{n}\right) s+\xi(s), \\
g(s) & =g(0)+\chi(s) .
\end{aligned}
$$

Note that

$$
\lim _{s \rightarrow 0^{+}} \frac{\xi(s)}{s}=0, \quad \lim _{s \rightarrow 0^{+}} \chi(s)=0 .
$$

Define a linear operator $A: D(A) \subset X \rightarrow X$

$$
(A u)(t)=-u^{\prime}(t)+a(t) g(0) u(t), \quad u \in D(A),
$$

with

$$
D(A)=\left\{u \in C^{1}[0, \omega] \mid u \text { is } \omega \text {-periodic. }\right\} .
$$

From [18], it follows that $A^{-1}: X \rightarrow X$ is compact and continuous.

Now (43) can be rewritten to the form

$$
\begin{aligned}
u(t)= & \lambda A^{-1}\left[h(\cdot)\left(f^{[n]}\right)_{0} u(\cdot-\tau(\cdot))\right](t) \\
& +A^{-1} N(\lambda, u(\cdot))(t),
\end{aligned}
$$


where

$$
\begin{aligned}
N(\lambda, u(\cdot))(t):= & {[a(\cdot) \chi(u(\cdot)) u(\cdot)} \\
& -\lambda h(\cdot) \xi(u(\cdot-\tau(\cdot)))](t) .
\end{aligned}
$$

It is easy to check that

$$
\lim _{\|u\| \rightarrow 0} \frac{N(\lambda, u)}{\|u\|}=0, \quad \text { uniformly on bounded } \lambda \text { intervals. }
$$

It is easy to check that (48) is equivalent to

$$
A u=\lambda h(\cdot)\left(f^{[n]}\right)_{0} u(\cdot-\tau(\cdot))(t)+N(\lambda, u(\cdot))(t) .
$$

Let us consider (48) as a bifurcation problem from the trivial solution $u \equiv 0$.

Since $\left(f^{[n]}\right)_{0}>0$, the results of Nonlinear Krein-Rutman Theorem (see Dancer [22] and Zeidler [23, Corollary 15.12]) for (48) can be stated as follows: there exists a continuum $\mathscr{C}_{+}^{[n]}$ of positive solutions of (48) joining $\left(\lambda^{\diamond}(g(0)) /\left(f^{[n]}\right)_{0}, 0\right)$ to infinity in $K$. Moreover, $\mathscr{C}_{+}^{[n]} \backslash\left\{\left(\lambda^{\diamond}(g(0)) /\left(f^{[n]}\right)_{0}, 0\right)\right\} \subset$ int $K$ and $\left(\lambda^{\diamond}(g(0)) /\left(f^{[n]}\right)_{0}, 0\right)$ is the only bifurcation point of (48) lying on trivial solutions line $u \equiv 0$.

Proof of Theorem 6. Let us verify that $\left\{\mathscr{C}_{+}^{[n]}\right\}$ satisfies all of the conditions of Theorem 2.

It follows from (42) that $\lambda^{\diamond}(g(0)) /\left(f^{[n]}\right)_{0}=: \eta_{n} \rightarrow 0$ as $n \rightarrow \infty$. Therefore, (A2) holds.

Let $r>0$ be fixed. Then there exists $n_{0} \in \mathbb{N}$, such that

$$
\frac{1}{n} \leq \delta r .
$$

Thus

$$
M_{r}(f)=M_{r}\left(f^{[n]}\right), \quad n \geq n_{0} .
$$

From this and Lemma 9, it follows that there exists $\lambda_{*}(r)$ with $0<\lambda_{*}(r)<r\left(1-\sigma^{l}\right) / M_{r} \int_{0}^{\omega} h(s) d s$, such that (48) has no solution $(\lambda, u)$ with

$$
\|u\|=r, \quad 0<\lambda<\lambda_{*}(r) .
$$

Since $r$ is arbitrary, we see that (A1) is satisfied.

(A3) can be deduced directly from the Arzela-Ascoli Theorem and the definition of $f^{[n]}$.

Therefore, the superior limit of $\left\{\mathscr{C}_{+}^{[n]}\right\}$ contains an unbounded component $\mathscr{C}$ with $(0,0) \in \mathscr{C}$.

Moreover, $\mathscr{C} \cap\left\{(\mu, y) \mid 0<\mu \leq \lambda_{*}(r),\|y\|=r\right\}=\emptyset$.

Finally, we show that $\mathscr{C}$ meets $(0,0)$ and $(0, \infty)$.

Let $\left\{\mu_{n}, y_{n}\right\} \subset \mathscr{C}$ with

$$
\left|\mu_{n}\right|+\left\|y_{n}\right\| \rightarrow \infty .
$$

We claim that $\mu_{n} \rightarrow 0^{+}$.

Assume on the contrary that $\mu_{n} \rightarrow+\infty$. Let

$$
v_{n}(t)=\frac{y_{n}(t)}{\left\|y_{n}\right\|} .
$$

Then

$$
\begin{gathered}
v_{n}^{\prime}(t)=a(t) g\left(y_{n}(t)\right) v_{n}(t)-\mu_{n} h(t) \\
\times \frac{f\left(y_{n}(t-\tau(t))\right)}{y_{n}(t-\tau(t))} v_{n}(t-\tau(t)), \quad t \in \mathbb{R}, \\
v_{n} \in X .
\end{gathered}
$$

So $v_{n}^{\prime}(t)<0$ on $[0, \omega]$ as $n \rightarrow \infty$. This contradicts (58). Thus (55) implies that

$$
\left\|y_{n}\right\| \longrightarrow \infty
$$

Assume on the contrary that $\mu_{n} \rightarrow \alpha>0$ (after taking a subsequence and relabeling if necessary).

Since $y_{n}(t) \geq \delta\left\|y_{n}\right\|$ on $[0, \omega]$, it follows from (59) that $y_{n}(t) \rightarrow \infty$ uniformly on $[0, \omega]$. This together with $f_{\infty}=\infty$ implies $v_{n}^{\prime}(t)<0$ on $[0, \omega]$ as $n \rightarrow \infty$. This contradicts (58) again.

Theorem 10. Let (C1)-(C2) hold. Assume that

(C4) $f_{0}=0 ; f_{\infty}=0$.

Then $\Sigma$ contains a component $\mathscr{C}$ satisfying the following.

(1) For given $\widehat{b}>0$,

$$
\sup \left\{\lambda \mid(\lambda, u) \in\left(\mathscr{C} \cap \Omega_{\widehat{b}, 1}\right)\right\}=+\infty .
$$

(2) For every $r>0$, there exists $\eta(r)>0$, such that

$$
\mathscr{C} \cap\{(\mu, u) \in[\mu(r), \infty) \times X \mid\|u\|=r\}=\emptyset .
$$

Corollary 11. Let (C1)-(C2) and (C4) hold. Then there is a constant $\eta^{*}>0$ such that (24) has at least two positive solutions as $\lambda>\eta^{*}$.

To prove Theorem 10, we define $f^{[n]}$ as in (40). Notice that (C4) implies that

$$
\lim _{n \rightarrow \infty}\left(f^{[n]}\right)_{0}=0 .
$$

Let $\xi, \chi$ be the function satisfying (44)-(45).

Now (43) can be rewritten to the form

$$
\begin{aligned}
u(t)= & \lambda A^{-1}\left[h(\cdot)\left(f^{[n]}\right)_{0} u(\cdot-\tau(\cdot))\right](t) \\
& +A^{-1} N(\lambda, u(\cdot))(t),
\end{aligned}
$$

where

$$
\begin{aligned}
N(\lambda, u(\cdot))(t):= & {[a(\cdot) \chi(u(\cdot)) u(\cdot)} \\
& -\lambda h(\cdot) \xi(u(\cdot-\tau(\cdot)))](t) .
\end{aligned}
$$

It is easy to check that

$$
\lim _{\|u\| \rightarrow 0} \frac{N(\lambda, u)}{\|u\|}=0, \quad \text { uniformly on bounded } \lambda \text { intervals. }
$$


Let us consider (63) as a bifurcation problem from the trivial solution $u \equiv 0$.

By similar method to deal with (48) in which $f_{0}=\infty$, we have from $\left(f^{[n]}\right)_{0}>0$ and [23, Corollary 15.12]) that there exists a continuum $\mathscr{C}_{+}^{[n]}$ of positive solutions of (63) joining $\left(\lambda^{\diamond}(g(0)) /\left(f^{[n]}\right)_{0}, 0\right)$ to infinity in $K$. Moreover, $\mathscr{C}_{+}^{[n]} \backslash$ $\left\{\left(\lambda^{\diamond}(g(0)) /\left(f^{[n]}\right)_{0}, 0\right)\right\} \subset$ int $K$ and $\left(\lambda^{\diamond}(g(0)) /\left(f^{[n]}\right)_{0}, 0\right)$ is the only bifurcation point of (48) lying on trivial solutions line $u \equiv 0$.

Lemma 12. Assume that (C1)-(C2) hold. If $u \in \partial K_{r}, r>0$, then

$$
\left\|T_{\lambda} u\right\| \geq \lambda \frac{\sigma^{L} \widehat{m}_{r}}{1-\sigma^{L}} \int_{0}^{\omega} h(s) d s,
$$

where

$$
\widehat{m}_{r}=\min _{\delta r \leq x \leq r}\{f(x)\} .
$$

Proof. Since $\sigma^{L} /\left(1-\sigma^{L}\right) \leq G_{u}(t, s)$ and $f(u(t)) \geq \widehat{m}_{r}$ for $t \epsilon$ $[0, \omega]$, it follows that

$$
\begin{aligned}
\left\|T_{\lambda} u\right\| & \geq \lambda \int_{t}^{t+\omega} G_{u}(t, s) h(s) f(u(s)) d s \\
& \geq \lambda \frac{\sigma^{L} \widehat{m}_{r}}{1-\sigma^{L}} \int_{t}^{t+\omega} h(s) d s \geq \lambda \frac{\sigma^{L} \widehat{m}_{r}}{1-\sigma^{L}} \int_{0}^{\omega} h(s) d s .
\end{aligned}
$$

Lemma 13. Assume that (C1)-(C2) and (C4) hold, and let I be a compact subinterval of $(0, \infty)$. Then

$$
\|u\| \leq M_{I},(\lambda, u) \in\left\{(\lambda, u) \in C_{+}^{[n]} \mid \lambda \in I\right\}
$$

for some positive constant $M_{I}$, independent of $n, \lambda$, and $u$.

Proof. Assume on the contrary that there exists a sequence $\left\{\left(\mu_{k}, y_{k}\right)\right\} \subset \mathscr{C}_{+}^{[n]} \cap(I \times K)$ such that

$$
\left\|y_{k}\right\| \longrightarrow \infty
$$

Set $v_{k}(t)=y_{k}(t) /\left\|y_{k}\right\|$. Then

$$
\begin{gathered}
\left\|v_{k}\right\|=1, \\
v_{n}^{\prime}(t)=a(t) g\left(y_{n}(t)\right) v_{n}(t)-\mu_{n} h(t) \\
\times \frac{f\left(y_{n}(t-\tau(t))\right)}{y_{n}(t-\tau(t))} v_{n}(t-\tau(t)), \quad t \in \mathbb{R}, \\
v_{n} \in X .
\end{gathered}
$$

By (72), $\left\{v_{n}^{\prime}\right\}$ is bounded in $X$. This together with the fact that $\left\{\mu_{n}\right\} \subset I$ implies that there exists $\left(\mu_{*}, v_{*}\right) \in I \times X$ with

$$
\left\|v_{*}\right\|=1,
$$

such that

$$
\lim _{k \rightarrow \infty}\left(\mu_{k}, v_{k}\right)=\left(\mu_{*}, v_{*}\right), \quad \text { in } \mathbb{R} \times X
$$

(after choosing a subsequence and relabeling if necessary). Since $\left\{g\left(u_{n}\right)\right\}$ is bounded in $X,\left\{g\left(u_{n}\right)\right\}$ is bounded in $L^{2}(0, \omega)$, and subsequently, $g\left(u_{n}\right) \rightarrow \widehat{g}$ for some $\hat{g} \in L^{2}(0, \omega)$. By the standard method, we can prove that

$$
l \leq \widehat{g}(t) \leq L, \quad \text { a.e. } t \in[0, \omega]
$$

Moreover, combining (75) and (76) with the assumption $f_{\infty}=0$ and the corresponding integral equations of (72) and (73) and using Lebesgue dominated convergence theorem, we conclude that

$$
\begin{gathered}
v_{*}^{\prime}(t)-a(t) \widehat{g}(t) v^{*}=0, \quad \text { a.e. } t \in \mathbb{R}, \\
v_{*} \in X .
\end{gathered}
$$

Note that $a(t) \widehat{g}(t) v^{*} \geq 0$ and $a(t) \widehat{g}(t) v^{*} \quad \not \quad 0$ on $[0, \omega]$. This means that $v_{*}(0)<v_{*}(\omega)$. However, this contradicts (78).

Now, we are in the position to prove Theorem 10.

Proof of Theorem 10 (sketched). Fixed $r>0$. From Lemma 12, it follows that (63) has no solution if

$$
\lambda>\lambda^{*}(r)=: 1+\frac{r}{\left(\sigma^{L} \widehat{m}_{r} /\left(1-\sigma^{L}\right)\right) \int_{0}^{\omega} h(s) d s} .
$$

Applying Lemma 13, it is easy to verify that $\left\{\mathscr{C}_{+}^{[n]}\right\}$ satisfies all of the conditions of Theorem 3 . So, there exists a component $\mathscr{C}$ in $\varlimsup \mathscr{C}_{+}^{[n]}$ such that

(a) both $\mathscr{C} \cap \Omega_{\lambda^{*}(1), 1}$ and $\left(\mathscr{C} \cap\left(([a, \infty) \times X) \backslash \Omega_{\lambda^{*}(1), 1}\right)\right)$ are unbounded;

(b) $\mathscr{C} \cap\left\{(\mu, y) \mid \mu \geq \lambda^{*}(1),\|y\|=1\right\}=\emptyset$.

Lemma 13 guarantees that $\mathscr{C}$ satisfies

$$
\sup \left\{\lambda \mid(\lambda, u) \in\left(\mathscr{C} \cap \Omega_{\lambda^{*}(1), 1}\right)\right\}=+\infty \text {. }
$$

\section{Conflict of Interests}

The authors declare that there is no conflict of interests regarding the publication of this paper.

\section{Acknowledgments}

The authors are very grateful to the anonymous referees for their valuable suggestions. The research was supported by the NSFC (nos. 11301059 and 11361054) and HSSF of Ministry of Education of China (no. 13YJA790078). 


\section{References}

[1] J. Leray and J. Schauder, "Topologie et équations fonctionnelles," Annales Scientifiques de l'École Normale Supérieure, vol. 51, pp. 45-78, 1934.

[2] F. E. Browder, "On continuity of fixed points under deformations of continuous mappings," Summa Brasiliensis Mathematicae, vol. 4, pp. 183-191, 1960.

[3] D. G. Costa and J. V. A. Gonçalves, "Existence and multiplicity results for a class of nonlinear elliptic boundary value problems at resonance," Journal of Mathematical Analysis and Applications, vol. 84, no. 2, pp. 328-337, 1981.

[4] I. Massabò and J. Pejsachowicz, "On the connectivity properties of the solution set of parametrized families of compact vector fields," Journal of Functional Analysis, vol. 59, no. 2, pp. 151-166, 1984.

[5] J. Sun and F. Song, "A property of connected components and its applications," Topology and Its Applications, vol. 125, no. 3, pp. 553-560, 2002.

[6] G. T. Whyburn, Topological Analysis, Princeton University Press, 1958.

[7] R. Ma and Y. An, "Global structure of positive solutions for superlinear second order $m$-point boundary value problems," Topological Methods in Nonlinear Analysis, vol. 34, no. 2, pp. 279-290, 2009.

[8] W. S. C. Gurney, S. P. Blythe, and R. M. Nisbet, "Nicholson's blowflies revisited," Nature, vol. 287, no. 5777, pp. 17-21, 1980.

[9] M. C. Mackey and L. Glass, "Oscillation and chaos in physiological control systems," Science, vol. 197, no. 4300, pp. 287-289, 1977.

[10] M. Ważewska-Czyżewska and A. Lasota, "Mathematical problems of the dynamics of a system of red blood cells," Matematyka Stosowana, vol. 6, pp. 23-40, 1976 (Polish).

[11] S.-N. Chow, "Existence of periodic solutions of autonomous functional differential equations," Journal of Differential Equations, vol. 15, pp. 350-378, 1974.

[12] H. I. Freedman and J. H. Wu, "Periodic solutions of singlespecies models with periodic delay," SIAM Journal on Mathematical Analysis, vol. 23, no. 3, pp. 689-701, 1992.

[13] J. Wu and Z. Wang, "Positive periodic solutions of second-order nonlinear differential systems with two parameters," Computers \& Mathematics with Applications, vol. 56, no. 1, pp. 43-54, 2008.

[14] S. S. Cheng and G. Zhang, "Existence of positive periodic solutions for non-autonomous functional differential equations," Electronic Journal of Differential Equations, vol. 59, pp. 1-8, 2001.

[15] D. Q. Jiang and J. J. Wei, "Existence of positive periodic solutions for nonautonomous delay differential equations," Chinese Annals of Mathematics A, vol. 20, no. 6, pp. 715-720, 1999 (Chinese).

[16] D. Ye, M. Fan, and H. Wang, "Periodic solutions for scalar functional differential equations," Nonlinear Analysis: Theory, Methods \& Applications, vol. 62, no. 7, pp. 1157-1181, 2005.

[17] Y. Li, X. Fan, and L. Zhao, "Positive periodic solutions of functional differential equations with impulses and a parameter," Computers \& Mathematics with Applications, vol. 56, no. 10, pp. 2556-2560, 2008.

[18] H. Wang, "Positive periodic solutions of functional differential equations," Journal of Differential Equations, vol. 202, no. 2, pp. 354-366, 2004.

[19] Z.-L. Jin and H. Wang, "A note on positive periodic solutions of delayed differential equations," Applied Mathematics Letters, vol. 23, no. 5, pp. 581-584, 2010.
[20] K. Deimling, Nonlinear Functional Analysis, Springer, Berlin, Germany, 1985.

[21] D. J. Guo and V. Lakshmikantham, Nonlinear Problems in Abstract Cones, Academic Press, Orlando, Fla, USA, 1988.

[22] E. Dancer, "Global solutions branches for positive maps," Archive for Rational Mechanics and Analysis, vol. 55, no. 3, pp. 207-213, 1974.

[23] E. Zeidler, Nonlinear Functional Analysis and Its Applications. I. Fixed-Point Theorems, Springer, New York, NY, USA, 1986. 


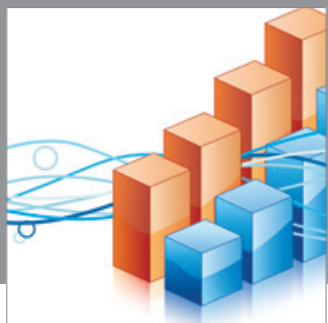

Advances in

Operations Research

mansans

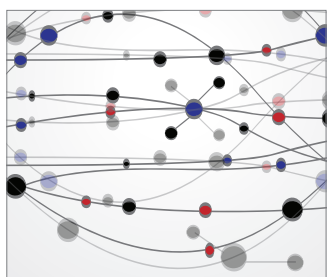

The Scientific World Journal
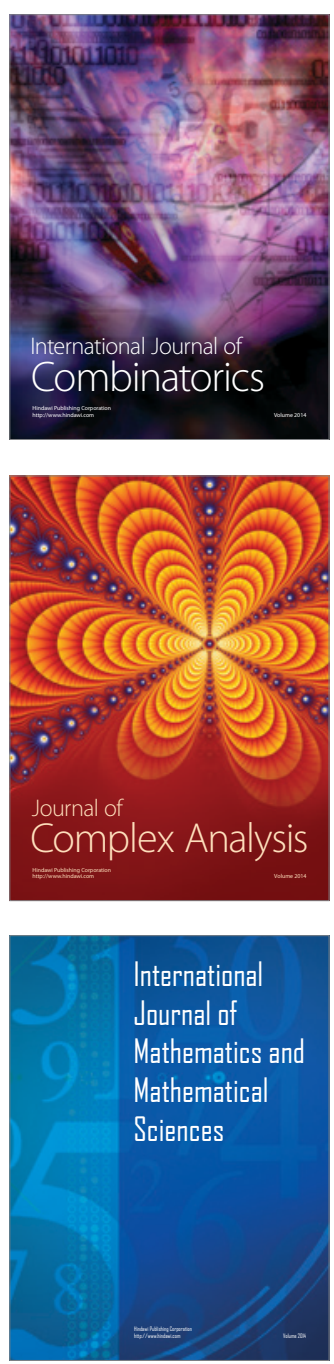
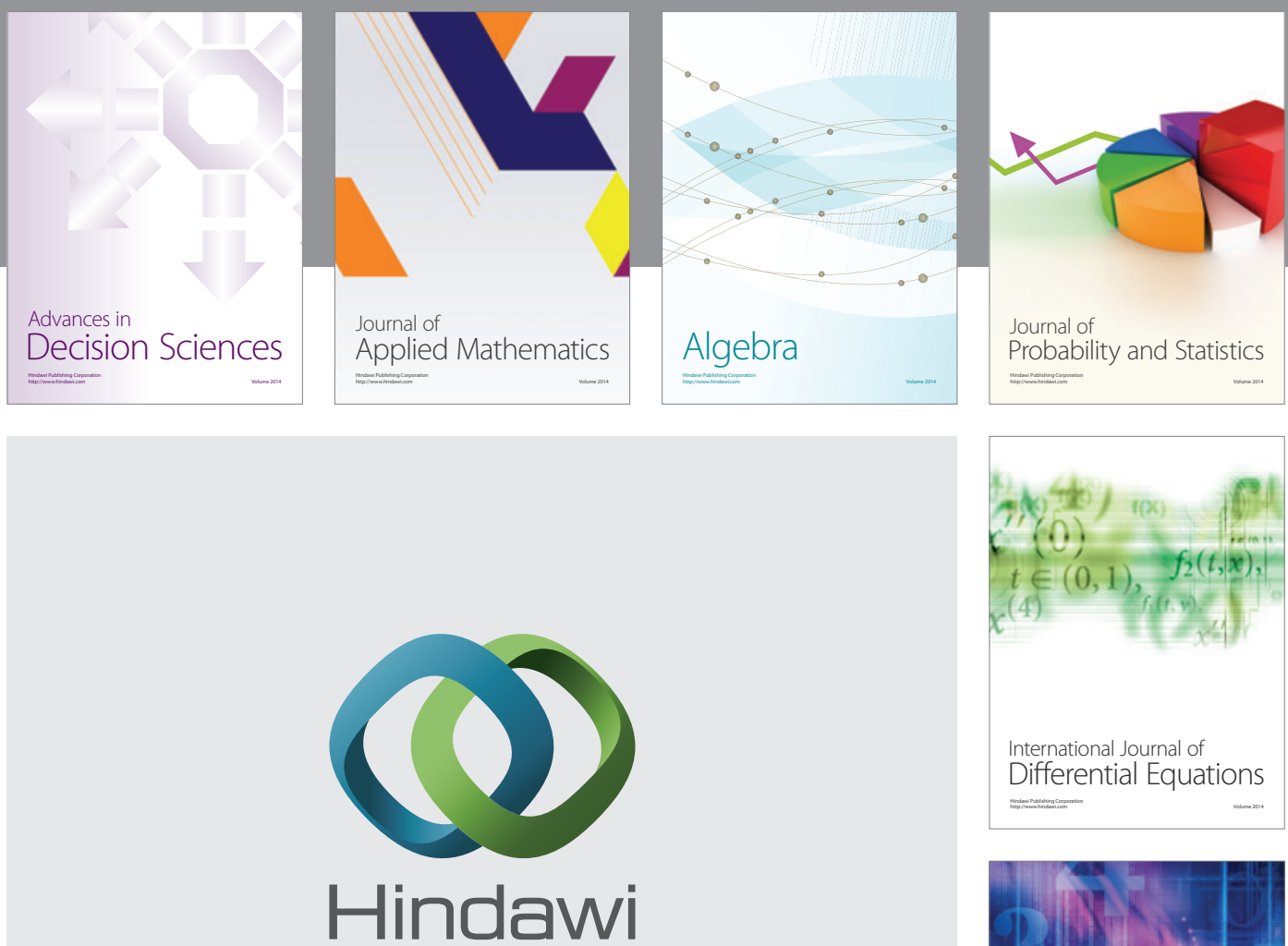

Submit your manuscripts at http://www.hindawi.com
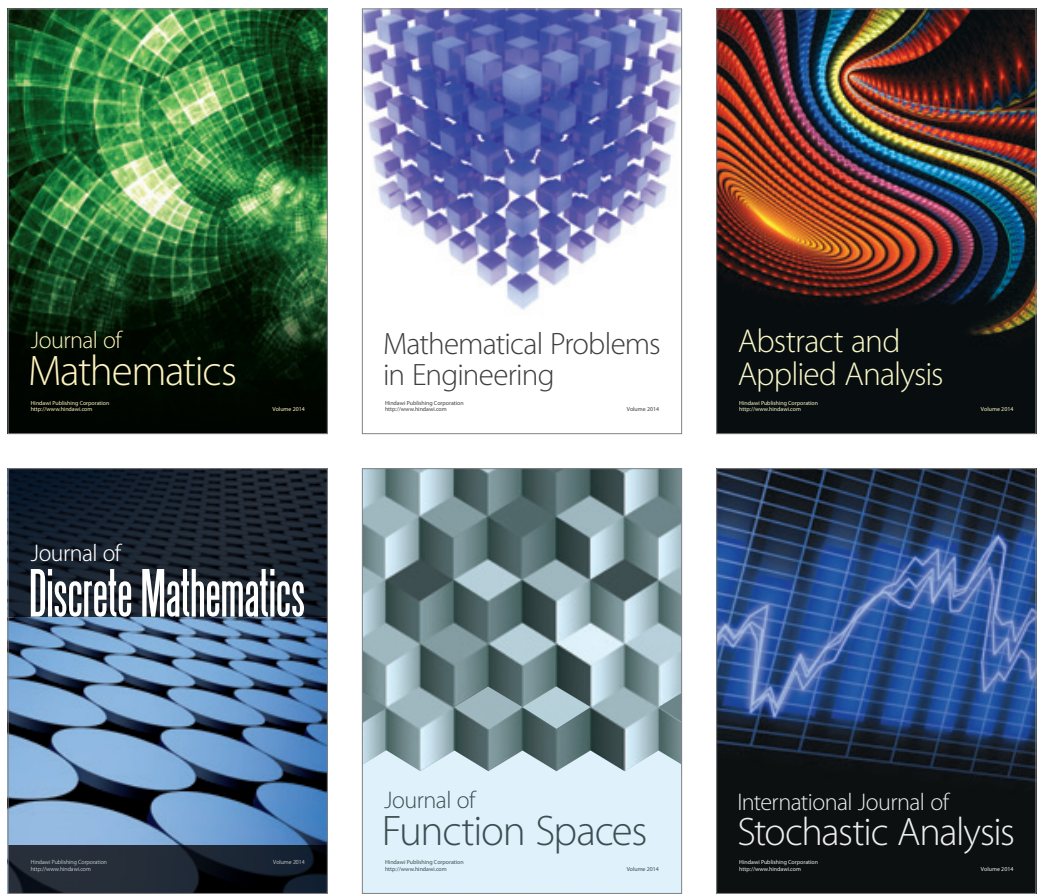

Journal of

Function Spaces

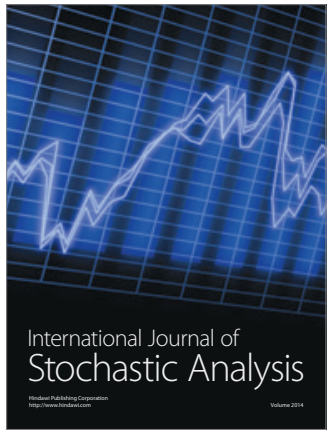

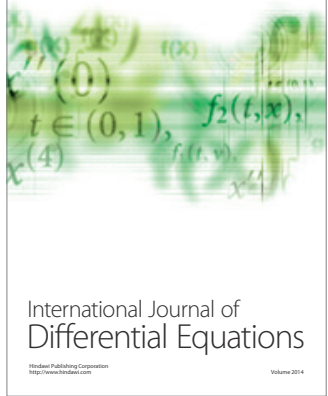
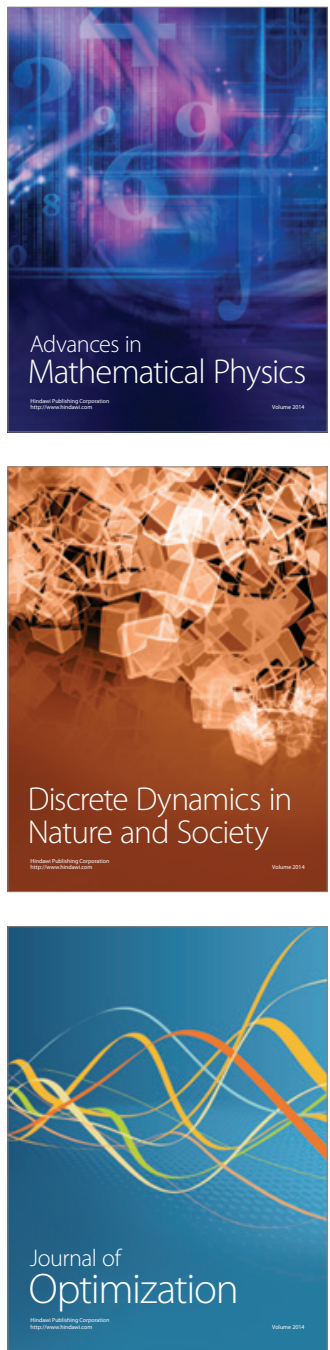